\title{
26. NONGRAVITATIONAL EFFECTS ON COMETS: THE CURRENT STATUS
}

\author{
B. G. MARSDEN \\ Smithsonian Astrophysical Observatory, Cambridge, Mass., U.S.A.
}

\begin{abstract}
A method for allowing for the effects of nongravitational forces on the motions of comets is summarized. Study of the motions of specific comets indicates that these forces act essentially continuously but have a high inverse dependence on heliocentric distance; there is also evidence for secular changes. The equations of motion employed are discussed in terms of the Whipple icyconglomerate model. Nongravitational parameters are tabulated for all 46 comets observed at three or more perihelion passages. We point out the particular problems that still exist for certain comets and suggest directions for future research.
\end{abstract}

Few astronomers would deny that comets are subjected to forces of a nongravitational nature: one has merely to consider the many instances where comets have split or have exhibited great surges in brightness. There has been severe disagreement, however, as to whether nongravitational forces have detectable effects on the motions of comets. Controversy has raged ever since Encke first claimed, a century and a half ago, that such effects were present in the motion of the comet that bears his name, and similar claims made during the second half of the nineteenth century and the first half of the twentieth with respect to a number of other comets have failed to clarify the situation.

The conservative element has pointed out, with considerable justification, that conclusions regarding the orbits of comets can be influenced, not only by possible systematic errors in the observations of comets, but also - and particularly - by errors in the computations themselves (Roemer, 1961). Even in the most sophisticated computations approximations have been introduced, and there was no way of knowing how the resulting errors would propagate when a comet was moderately perturbed by Jupiter, for example. The partial derivatives used for the differential correction of the orbit were invariably calculated from the traditional Keplerian expressions (e.g., Eckert and Brouwer, 1937), whereas they should be the derivatives of the instantaneous, perturbed coordinates with respect to the osculating elements at some specified epoch. The easiest and surest way of obtaining correct partial derivatives is to calculate the perturbations, not only on the preliminary orbit, but also on the six orbits obtained by varying each element one by one by a small amount; the derivatives are then taken to be the differences between the residuals from the preliminary orbit and from each variation orbit in turn. The process should be iterated until it has converged to the requisite number of figures.

In the days of logarithms and desk-calculators it was clearly impractical to try to fit rigorously the gravitational orbit of a periodic comet to observations at several apparitions. Now that high-speed computers can be used this is no longer the case, and the rigorous calculations made during the last few years for a number of comets, 
including the perturbations by all the principal planets, show quite conclusively that, as a general rule, unacceptable systematic trends remain in the residuals (Marsden, 1968). These systematic trends become larger the longer the interval of time covered by the observations used, and they sometimes amount to several minutes of arc over only three apparitions. Since the possible departure of the center of mass of a comet from the center of light is not more than a few seconds of arc - for modern photographic observations with long-focus reflectors at any rate - it is quite clear that additional forces are involved. The failure to fit a gravitational orbit is most extreme for a comet such as $\mathrm{P} / \mathrm{d}$ 'Arrest or $\mathrm{P} /$ Honda-Mrkos-Pajdušáková, where any condensation is hard to detect in the midst of the large coma; on the other hand, comets like $\mathrm{P} /$ Arend-Rigaux and $\mathrm{P} /$ Neujmin 1 , which have nearly always been indistinguishable from minor planets in appearance, are found to conform to purely gravitational theory.

Another controversy, and one not so easily resolved, concerns whether the nongravitational forces take the form of discrete impulses or whether they act more or less continuously. The most readily detectable nongravitational effect is, of course, the progressive advance or delay of a comet at successive returns to perihelion, and the standard way to allow for this has been to postulate a secular variation in the mean motion. Studies made in this manner - affected though they might have been by approximations - indicated that for a particular comet this variation was surprisingly regular, which is a strong point in favor of the hypothesis that the forces act continuously. And attempts to relate nongravitational effects in the motion of a comet to direct observations of the influence of nongravitational forces on the comet's physical appearance have failed miserably: according to the calculations by Cunningham (1968) and by Herget (1968) P/Schwassmann-Wachmann 1 - a comet that quite frequently throws off shells of matter and suddenly increases in brightness a hundredfold shows in its motion no nongravitational effects whatsoever.

Accordingly, we have supposed the nongravitational forces to act continuously and have included in the cometary equations of motion additional acceleration components $F_{1}, F_{2}$ and $F_{3}$, where $F_{1}$ is directed outward along the radius vector, $F_{2}$ is directed parallel to the line from the Sun to the point in the orbit with true anomaly $90^{\circ}$ ahead of the comet (i.e., approximately along the velocity vector in the case of low orbital eccentricity), and $F_{3}$ is directed perpendicular to the orbit plane and such that one has a right-handed system (i.e., it is toward the north pole of the orbit). It is reasonable that the $F_{i}$ should depend on the comet's heliocentric distance $r$, and while it is not possible to determine this dependence precisely, we have found that in general it is considerably more extreme than an inverse square or inverse cube. For definiteness, but certainly not uniqueness, we have generally adopted the form

$$
F_{i}=G_{i} r^{-3} \exp \left(-r^{2} / 2\right)
$$

where $r$ is measured in AU.

The orbit of a comet can thus be differentially corrected, not only for the usual six elements, but also for the three nongravitational parameters $G_{1}, G_{2}$ and $G_{3}$, 
assumed constant. The additional partial derivatives may be formed numerically in precisely the manner indicated for the others. Whenever we have solved for $G_{3}$ we have found it to be determined to less than its mean error, so the solution may be limited to the nongravitational components in the comet's orbit plane. Such solutions have been made to date for 16 periodic comets (e.g., Marsden, 1969, 1970). The transverse component $G_{2}$ is related rather closely to the secular variation in the comet's mean motion and is sometimes determined to several hundred times its mean error. However, the radial component $G_{1}$ can often be determined surprisingly well too, occasionally to several tens of times its mean error.

In terms of Whipple's (1950) icy-conglomerate model for a cometary nucleus (assumed spherical) the $G_{i}$ are:

$$
\begin{aligned}
G_{1}= & \xi\left[\cos \lambda\left(1-\frac{1}{2} \sin ^{2} I\right)\right. \\
& \left.+\frac{1}{2} \sin ^{2} I-\frac{1}{2}(1-\cos \lambda) \sin ^{2} I \cos (2 \varphi+2 f)\right] \\
G_{2}= & \xi\left[\sin \lambda \cos I+\frac{1}{2}(1-\cos \lambda) \sin ^{2} I \sin (2 \varphi+2 f)\right] \\
G_{3}= & \xi \sin I[\sin \lambda \cos (\varphi+f)-(1-\cos \lambda) \cos I \sin (\varphi+f)],
\end{aligned}
$$

where $f$ is the comet's true anomaly, $\varphi$ the longitude of the meridian of the comet facing the Sun at the comet's passage through perihelion, $I$ the inclination of the comet's equator to its orbit, $\lambda(\geqslant 0)$ the lag-angle of the direction of maximum mass ejection behind the subsolar meridian, and $\xi(\geqslant 0)$ gives the magnitude of the reactive force on the comet.

If we make the usual assumption that $\sin I=0$, Equations (2) become, simply,

$$
\begin{aligned}
& G_{1}=\xi \cos \lambda \\
& G_{2}= \pm \xi \sin \lambda \\
& G_{3}=0,
\end{aligned}
$$

the choice of sign depending on whether $I=0$ or $180^{\circ}$. When $G_{1}$ is well determined we have found it to be positive, showing that the radial component of the force acts away from the Sun, and particularly for comets of perihelion distance $q$ greater than about 1.4 $\mathrm{AU} G_{1}$ is an order of magnitude greater than $G_{2}$. This suggests that, in general, $\lambda$ is a small and thus approximately constant angle in the first quadrant and that the sign of $G_{2}$ corresponds directly to the choice $I=0$ or $180^{\circ}$. This is perhaps what one would expect for a 'new' comet, a 'clean snowball' that has only recently been perturbed by Jupiter into an orbit of relatively small $q$. On the other hand, although the solutions are not completely satisfactory, there are indications that $G_{1}$ may be slightly negative for $\mathrm{P} /$ Encke and $\mathrm{P} /$ Pons-Winnecke. But we know that these comets are 'old' and that they have existed for some time with $q$ less than $0.8 \mathrm{AU}$ (only $0.3 \mathrm{AU}$ for P/Encke); their nuclear constructions must now be extremely complex, and we cannot expect the simple Equations (3), or even Equations (2), to apply.

Solutions for constant $G_{1}$ and $G_{2}$, as well as the six orbital constants, greatly extend the interval over which one can obtain a satisfactory representation of the 
orbit of a periodic comet. However, as discovered a century ago by Asten and Backlund in the case of $\mathrm{P} /$ Encke, the nongravitational effects do not completely reproduce themselves every revolution. Asten, Backlund, and more recently Makover and others at the Institute for Theoretical Astronomy here in Leningrad have found that the secular variation in the mean motion of $\mathrm{P} /$ Encke has been decreasing. This suggests that when solutions for constant $G_{1}$ and $G_{2}$ cease to be satisfactory we might consider these quantities to vary in some regular way with the time. In order to avoid the possibility that $G_{2}$ for P/Encke would change sign in the near future and that it would then rapidly increase in magnitude - which does not seem physically to be very probable - we have adopted the exponential variation

$$
G_{i}=A_{i} \exp \left(-B_{i} \tau\right)
$$

where the $A_{i}$ and $B_{i}$ are now constants, and $\tau$ is the time from an initial epoch (measured, for convenience, in units of $10^{4}$ days, or approximately $27.4 \mathrm{yr}$ ).

We have not found it useful to solve separately for the radial variation $B_{1}$ (for this does not significantly affect the residuals) and have generally taken it to be zero. And since $A_{2}$ and $B_{2}$ are rather highly correlated we have solved for $B_{2}$ only if it produces a substantial improvement in the residuals; even then the results for $B_{2}$ should be regarded with a certain amount of caution. A solution for $\mathrm{P} /$ Encke, fitted to observations over the interval 1927-1967, gave $B_{2}=+0.8$ (implying that $G_{2}$ decreases with a half-life of $36 \mathrm{yr}$ ). Extrapolation back to the early nineteenth century, however, would make the nongravitational force much larger than was observed, and we must suppose that $B_{2}$ has steadily increased from the value then of +0.3 . Four other comets, notably the well-observed P/Schwassmann-Wachmann 2 , have given positive values of $B_{2}$, and indeed this is to be expected if the nongravitational effect eventually all but vanishes, and we are left with a comet like P/Arend-Rigaux and P/Neujmin 1 that appears to have lost practically all its volatile material.

With the use of the additional parameters $A_{1}$ and $A_{2}$, and if necessary also $B_{2}$, it is sometimes possible to represent the observations of a short-period comet at as many as six apparitions and to predict the comet's position with moderate accuracy at several more. Occasionally, however, a comet will appear far from its expected place. The most celebrated example of this is P/Perrine-Mrkos at its return in 1968. Sitarski (1968) had provided a careful, but purely Newtonian, prediction based on the observations in 1955 and 1961-1962. The comet had also been observed in 1909 and 1896-1897, and extrapolation back suggested that nongravitational effects would advance $T$ in 1968 by 0.10 day, a relatively large correction but by no means unusual. In actual fact $T$ was advanced by an additional 0.7 day, and since the comet was rather near the Earth, the error in the geocentric position was well over $2^{\circ}$; the error along the comet's orbit was almost $2000000 \mathrm{~km}$. We surmised that the reason for the discrepancy stemmed from the fact that the comet passed only 0.4 AU from Jupiter in 1959, the resulting orbital change altering the pattern of solar radiation on the comet, which in turn affected the nongravitational parameters. The anomaly could be considered as a sudden decrease of $3.5 \mathrm{~m} \mathrm{~s}^{-1}$ in velocity when the comet was near Jupiter. 
There is also evidence that the same phenomenon, though to a lesser extent, occurred in the case of $\mathrm{P} /$ Schaumasse around the time of its approach to Jupiter (also to 0.4 $\mathrm{AU})$ in 1937. On the other hand several more comets, even comets on which the nongravitational effects are normally large, have passed near Jupiter and subsequently been perfectly predictable. A noteworthy example is $\mathrm{P} / \mathrm{d}$ 'Arrest, the orbit fitted to observations over 1923-1964 requiring $\Delta T$ corrections of only some 0.02 day in 1910 and in 1970, this in spite of approaches to Jupiter of less than $0.5 \mathrm{AU}$ in 1920 and 1968. And we don't know for sure that the troubles with P/Perrine-Mrkos and $\mathrm{P} /$ Schaumasse arose during the revolutions in which there were approaches to Jupiter - they might have arisen during the neighboring revolutions. Yeomans (1972) has conclusively shown that a large anomaly occurred in the motion of P/GiacobiniZinner between 1959 and 1965, an interval that did not involve an approach to Jupiter.

Even if we ignore the most recent apparition Yeomans' results on P/GiacobiniZinner show quite definitely that $B_{2}$ is negative. A somewhat less certain, but possibly more negative, $B_{2}$ has now been obtained for $\mathrm{P} /$ Honda-Mrkos-Pajdušáková. So the simple picture of the nongravitational effects slowly decreasing as a comet loses its volatiles cannot be correct. Furthermore, solutions for $A_{1}$ and $A_{2}$ over discrete arcs of observations of $\mathrm{P} /$ Pons-Winnecke and $\mathrm{P} /$ Faye have shown that $G_{2}$ can change sign. The change of sign for $\mathrm{P} / \mathrm{Pons}$-Winnecke between the nineteenth century and the present is very definite, but in the meantime the comet repeatedly made close approaches to Jupiter, and among the orbital changes there was a net increase of $50 \%$ in $q$. The nongravitational effects on $\mathrm{P} / \mathrm{Faye}$ are smaller and not so easily detectable, although the change of sign of $G_{2}$ also seems to be established; there have also been two moderately close approaches to Jupiter, but any modifications in the comet's orbit were minor.

Nevertheless, the values of $G_{2}$ for P/Pons-Winnecke and P/Faye are nowadays numerically smaller than they were, and while the change of sign means that we must abandon the straightforward exponential variation of Equation (4), it may be appropriate to modulate it with a periodic term; i.e., to adopt

$$
G_{i}=\exp \left(-B_{i} \tau\right)\left[A_{i}+D_{i} \cos \left(\beta_{i} \tau+\gamma_{i}\right)\right] \text {. }
$$

This form of damped oscillation was in fact adopted by Michielsen (1968) in a preliminary study of the secular acceleration of $\mathrm{P} /$ Encke. One could certainly assume that $D_{1}=0$, and solutions for all the quantities $A_{1}, A_{2}, B_{2}, D_{2}, \beta_{2}$, and $\gamma_{2}$ would only rarely be practical.

In modifying the equations that correspond to the Whipple model we made the assumption that $\sin I=0$. It followed from the observations that $\lambda$ is generally small. As an alternative, we could assume from the start that $\lambda$ is small but make no assumption about $I$. To an appropriate degree of approximation it follows that

$$
\begin{aligned}
G_{1} & =\xi \\
G_{2} & =\xi \lambda \cos I .
\end{aligned}
$$

$G_{3}$ is not now zero but would exhibit periodic variations with amplitude of order $\xi \lambda$. We certainly cannot exclude observationally the possibility that $G_{3}$ is of the same 
order as $G_{2}$, for $G_{3}$ is by no means as well determined as $G_{2}$; but by the same token it follows that $G_{3}$ can be ignored.

The important difference between Equations (3) and Equations (6) is the presence of the factor $\cos I$ in the latter. From a physical point of view nothing is known about the variation of $\cos I$ with time, but if there is a single dominant long-term variation, it is not unreasonable to associate this variation with the $D_{2}$ term in Equation (5). The $\exp \left(-B_{i} \tau\right)$ factor would be associated with the variation of $\xi$; and we should adopt $B_{1}=B_{2}$ and expect to find it positive. Gehrels (1970) has suggested that as a comet ages there may be a tendency for $I$ to stabilize at $90^{\circ}$, so oscillations of $I$ about $90^{\circ}$ and hence changes of sign of $G_{2}$ would not be uncommon in a comet's dying stages. There is a need for more theoretical, and if possible even experimental, work on the variations of $I$. It could be that large and sudden changes occur in $I$, and these may be responsible for the peculiar anomalies observed in the motions of $\mathrm{P} /$ PerrineMrkos, P/Schaumasse and P/Giacobini-Zinner.

In order to form the actual nongravitational acceleration components $F_{i}$ the quantities $G_{i}$ must be multiplied by some function of $r$. We found it convenient to define the relationship between the $F_{i}$ and $G_{i}$ by Equation (1). From studies of the periodic comets we know that an inverse square law is inappropriate for the transverse component, but it is not clear that an inverse square law is unsatisfactory in the case of the radial component. We could perhaps adopt an inverse square law for both components and then assume that the excess variation with heliocentric distance of $F_{2}$ is associated with the factor $\lambda$ in Equations (6); further theoretical study of the lagangle $\lambda$ is also most desirable.

There is some evidence that the radial component may indeed vary according to an inverse square law. The results discussed until now have been concerned with the short-period comets, and observations have to be made at three apparitions before the nongravitational effects show up. There are two recent long-period, single-apparition comets where it also appears to be possible to detect in their motions the effects of nongravitational forces. These are comets 1957 III (Arend-Roland) and 1960 II (Burnham), and a Newtonian orbit solution is particularly unsatisfactory in the latter case, even though the comet was under observation for only six months. For these comets it is the radial nongravitational component that is the better determined, and while there is some improvement if one defines it by Equation (1), the improvement is significantly greater if one supposes $F_{1}$ to vary simply according to $r^{-2}$. The repulsive force amounts to about $7 \times 10^{-5}$ that of solar gravitational attraction for comet 1957 III and to $20 \times 10^{-5}$ for comet 1960 II. These values are particularly high. Hamid and Whipple (1953) attempted to determine the radial nongravitational forces on 64 long-period comets by modifying the definitive orbit determinations. Most of their individual results are probably suspect, but their average result, a repulsion of about $1 \times 10^{-5}$ that of gravitational attraction by the Sun, could well be meaningful.

Our computations on the short-period comets suggest too that the radial repulsive force amounts to about $10^{-5}$ that of solar attraction. Equation (1) was adopted, but for both components the figures may be roughly converted to an effective inverse square law by dividing them by $q \exp \left(q^{2} / 2\right)$. The results so modified, and designated 
TABLE I

Nongravitational parameters for the 46 comets of three or more appearances

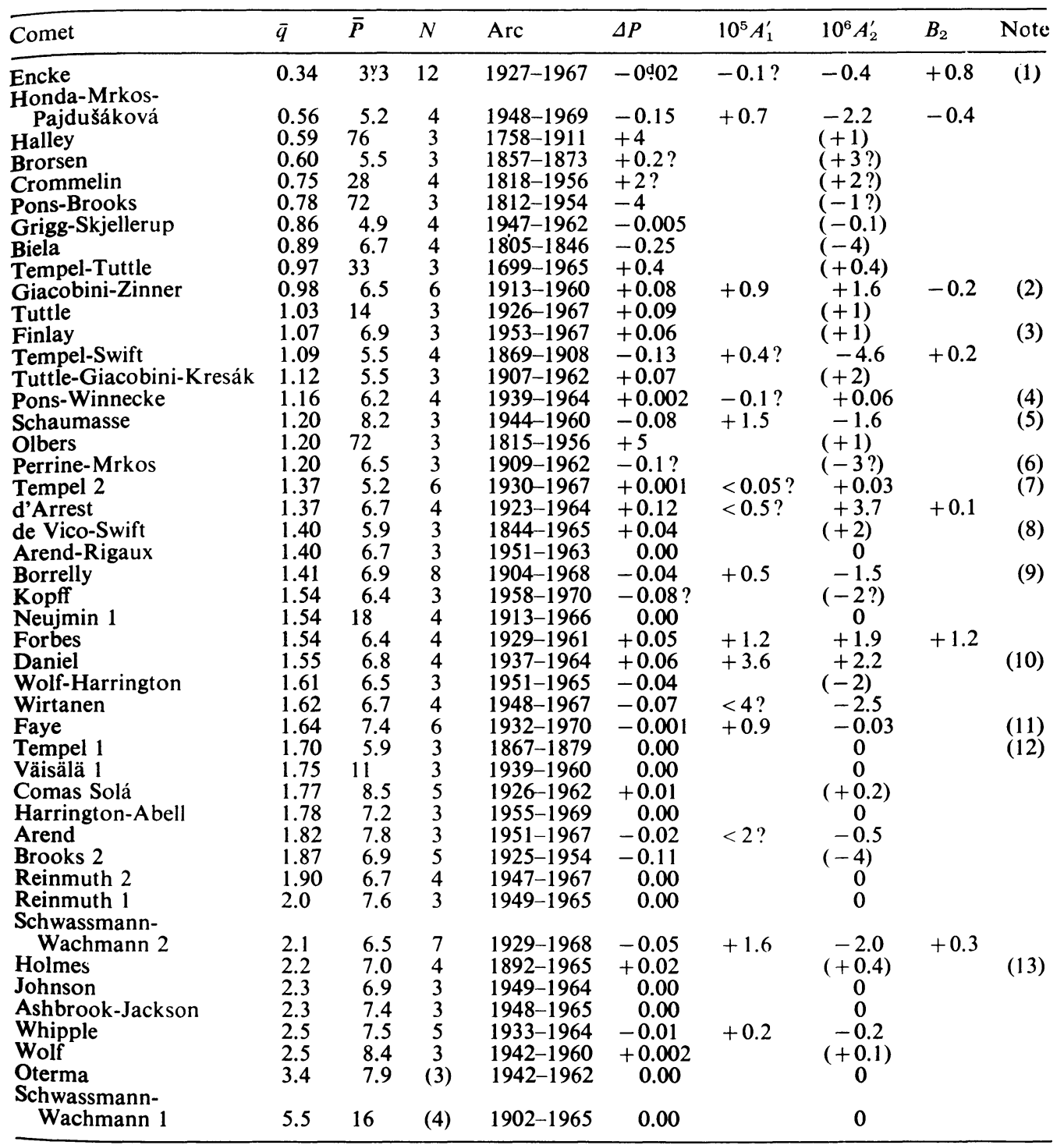

(1) $\mathrm{B}_{2}$ is evidently increasing and was only +0.3 in the early nineteenth century.

(2) In 1966 an additional correction $\Delta T=+0.3$ was required.

(3) Approach to $0.60 \mathrm{AU}$ of Jupiter in 1957.

(4) Five approaches within $0.7 \mathrm{AU}$ of Jupiter occurred every alternate revolution between 1882 and 1942. Before that $\Delta P\left(\right.$ and $A_{2}^{\prime}$ ) had the opposite sign. During 1858-1875 (when $\left.\bar{q}=0.79, \bar{P}=5.6\right) \Delta P=-0.02$, $10^{5} A_{2}^{\prime}=+0.6,10^{6} A_{2}^{\prime}=-0.4$.

(5) Approach to $0.37 \mathrm{AU}$ of Jupiter in 1937. In 1927 an additional correction $\Delta T=-0.4$ was required.

(6) Approach to $0.38 \mathrm{AU}$ of Jupiter in 1959. In 1968 an additional correction $\Delta T=-0.7$ was required.

(7) Approach to $0.63 \mathrm{AU}$ of Jupiter in 1943.

(8) Approaches to 0.60 AU of Jupiter in 1885 and to $0.44 \mathrm{AU}$ in 1897.

(9) Approach to $0.54 \mathrm{AU}$ of Jupiter in 1936.

(10) Approach to $0.53 \mathrm{AU}$ of Jupiter in 1959.

(11) $\Delta P$ (and $A_{2}^{\prime}$ ) formerly had the opposite sign. During $1888-1926$ (when $\bar{q}=1.69, \bar{P}=7.5$ ) $\Delta P=+0.01$, $10^{5} A_{1}^{\prime}=+0.9,10^{6} A_{2}^{\prime}++0.3$. Approaches to $0.51 \mathrm{AU}$ of Jupiter in 1899 and to $0.60 \mathrm{AU}$ in 1959 .

(12) Approach to $0.36 \mathrm{AU}$ of Jupiter in 1870.

(13) Approach to $0.54 \mathrm{AU}$ of Jupiter in 1908.

This table is based on calculations by K. Aksnes, J. L. Brady, M. P. Candy, H. J. Carr, A. D. Dubyago, J. Hepperger, P. Herget, M. Kamieński, L. Kresák, B. G. Marsden, J. G. Porter, E. K. Rabe, H. Q. 
by $A_{1}^{\prime}$ and $A_{2}^{\prime}$, are given in Table I, together with the values of $B_{2}$ and the effective period-change $\Delta P$, in days per period, due to the nongravitational forces. Rigorous computations of our nongravitational parameters have been made in relatively few cases, but $\Delta P$ values, though uncertain in many instances, can be estimated for all the comets of three or more apparitions. These $\Delta P$ values can be approximately converted into $A_{2}^{\prime}$ values, the results so obtained being given in parentheses in the table. The column $N$ gives the number of apparitions considered, and 'Arc' shows the actual span of the observations. The epoch for $B_{2}$ is the middle of this span. $\bar{P}$ denotes the mean period, and the comets are listed according to mean perihelion distance $\bar{q}$.

For only eleven of the entries are nongravitational forces completely undetectable, while positive and negative values of $\Delta P$ (and hence $A_{2}^{\prime}$ ) are equally numerous. The comets that show no detectable effects may perhaps be expected to show them when observations are available over longer intervals, but it should be noted that the two of smallest $\bar{q}$ are the two 'asteroidal' comets. Among the comets of $\bar{q}$ greater than about 1.9 AU small nongravitational effects prevail, and only P/SchwassmannWachmann 2 quite definitely shows them at all in fewer than four apparitions. Particularly noteworthy is the failure to detect nongravitational effects for the two comets of $\bar{q}$ greater than $2.5 \mathrm{AU}$, for low eccentricities have made it possible to observe these comets regularly even near aphelion, so their orbits are very well determined. It is perhaps to be expected that the motion of an icy comet would completely cease to be subject to nongravitational effects beyond a heliocentric distance of, say, 2.5 to 3.0 AU. Our continuous equations of motion, with the variation with $r$ given by Equation (1), simulate this but continue to give a nonzero contribution beyond the suggested cutoff. There would presumably be a more serious discrepancy if we were to adopt an inverse square law for $F_{1}$. P/Schwassmann-Wachmann 2 would thus not have been subject to nongravitational effects prior to its close approach to Jupiter in 1926, $q$ previously having been $3.5 \mathrm{AU}$. P/Brooks 2, another comet with large nongravitational effects, considering its present value of $q$, had a $q$ of $5.5 \mathrm{AU}$ until 1886. Long dormant, these comets have recently and suddenly become very active.

Although we have had a moderate amount of success in applying the procedures discussed here, it is by no means clear that we are really tackling the problem of nongravitational effects on cometary motions in the right manner. There is much room for further experimentation by a variety of methods, and the parentheses, blank spaces and question marks in Table I show that there are very many more comets left to be studied.

\section{References}

Cunningham, L. E.: 1968, private communication.

Eckert, W. J. and Brouwer, D.: 1937, Astron. J. 46, 125.

Gehrels, T.: 1970 , private communication.

Hamid, S. E. and Whipple, F. L.: 1953, Astron. J. 58, 100.

Herget, P.: 1968, Astron. J. 73, 729.

Marsden, B. G.: 1968, Astron. J. 73, 367. 
Marsden, B. G.: 1969, Astron. J. 74, 720.

Marsden, B. G.: 1970, Astron. J. 75, 75.

Michielson, H. F.: 1968, private communication.

Roemer, E.: 1961, Astron. J. 66, 368.

Sitarski, G.: 1968, Acta Astron. 18, 423.

Whipple, F. L.: 1950, Astrophys. J. 111, 375.

Yeomans, D. K.: 1972, this Symposium, p. 181.

\section{Discussion}

S. K. Vsekhsvyatskij: You speak of the continuous action of nongravitational forces. What is your opinion of the cases, studied by Sekanina, where cometary nuclei have split, the forces involved directly exhibiting their impulsive character?

B. G. Marsden: I am talking about a phenomenon that affects the motions of most, if not all, comets; and analysis of the orbit solutions suggests that it is continuous, inasmuch as anything is continuous. Of course, impulsive forces might yield observable effects in the case of a comet that has split.

L. Kresák: What is the period of the periodic term in your Equation (5) and how many cycles are covered by the observations of $\mathrm{P} /$ Encke?

B. G. Marsden: Michielsen obtained a period of something like 150 to $200 \mathrm{yr}$ for P/Encke barely a complete cycle since discovery. Calculations by Sekanina and myself suggest that the period may be similar in the case of $\mathrm{P} /$ Faye.

L. M. Shul'man: Outbursts in brightness are connected with symmetrical explosions in the nucleus. But the nucleus acquires almost zero momentum in this process, and we cannot expect any nongravitational forces.

B. G. Marsden: This is a good point, but are the outbursts of $\mathrm{P} / \mathrm{Sch}$ wassmann-Wachmann 1 sufficiently symmetrical for the forces to be cancelled out? 